\title{
Shallow-cloud impact on climate and uncertainty: A simple stochastic model
}

https://doi.org/10.1515/mcwf-2020-0002

Received September 22, 2019; accepted January 29, 2020

Abstract: Shallow clouds are a major source of uncertainty in climate predictions. Several different sources of the uncertainty are possible-e.g., from different models of shallow cloud behavior, which could produce differing predictions and ensemble spread within an ensemble of models, or from inherent, natural variability of shallow clouds. Here, the latter (inherent variability) is investigated, using a simple model of radiative statistical equilibrium, with oceanic and atmospheric boundary layer temperatures, $T_{o}$ and $T_{a}$, and with moisture $q$ and basic cloud processes. Stochastic variability is used to generate a statistical equilibrium with climate variability. The results show that the intrinsic variability of the climate is enhanced due to the presence of shallow clouds. In particular, the on-and-off switching of cloud formation and decay is a source of additional climate variability and uncertainty, beyond the variability of a cloud-free climate. Furthermore, a sharp transition in the mean climate occurs as environmental parameters are changed, and the sharp transition in the mean is also accompanied by a substantial enhancement of climate sensitivity and uncertainty. Two viewpoints of this behavior are described, based on bifurcations and phase transitions/statistical physics. The sharp regime transitions are associated with changes in several parameters, including cloud albedo and longwave absorptivity/carbon dioxide concentration, and the climate state transitions between a partially cloudy state and a state of full cloud cover like closed-cell stratocumulus clouds. Ideas of statistical physics can provide a conceptual perspective to link the climate state transitions, increased climate uncertainty, and other related behavior.

\section{Introduction}

Clouds have long been recognized as a leading source of uncertainty in future climate predictions $[5,7,9$, 41]. Furthermore, different cloud types can have different impacts. For example, to a certain degree, deep convective clouds have been seen to have a net radiative forcing that is less substantial than other cloud types, due to a near cancellation of their longwave and shortwave cloud-radiative feedbacks [15, 23, 33]. On the other hand, of particular importance for climate are shallow clouds, which can potentially have a substantial net cooling effect [6, 14].

A variety of simple models have helped to elucidate fundamental aspects of the climate system and water vapor and cloud feedbacks [2, 3, 20, 21, 25, 27, 32, 34, 35]. These models vary in their treatments of clouds and the hydrological cycle, and the climate system that is modeled is, for example, sometimes a particular region of deep convection or shallow convection and sometimes a two-box model that connects two such regions. Despite these and other differences, it is common in all of these models to treat climate circulations and cloud processes in idealized ways, and to analyze climate as a steady equilibrium.

*Corresponding Author: Eli A. Mueller: Stanford University, Department of Physics, 382 Via Pueblo Mall, Stanford,CA 943054060, E-mail: elim8@stanford.edu

Samuel N. Stechmann: Department of Mathematics, and Department of Atmospheric and Oceanic Sciences, University of Wisconsin-Madison, 480 Lincoln Dr, Madison, WI 53706, E-mail: stechmann@wisc.edu 
One of the main questions of interest in the present paper is: What is the uncertainty in the climate state due to the presence of shallow clouds? Such a question is motivated by the results of [6] and others, which suggest that marine boundary layer clouds are one of the main sources of uncertainty in predictions of climate and climate change. As a further question, one could ask: Is the uncertainty due to the use of an ensemble of models with different shallow cloud parameterizations, which might lead to different climate predictions from different models, or is it an inherent uncertainty from the natural variability of shallow clouds? The answer could potentially be a combination of these two effects, and a full answer is beyond the scope of the present paper. In the present paper, the aim is to focus on the latter: to investigate a simple model of shallow clouds and climate, and to assess the impact of shallow clouds on the model's "natural" variability. One particular model will be investigated in detail here, and, since the answer could potentially depend on the particular model, some extensions are also discussed in section 6.

It is difficult to investigate the question of uncertainty and variability by using steady equilibrium models described in a paragraph above. While one could investigate uncertainty with respect to model parameters using a steady equilibrium model, a dynamically evolving model is a natural setting to investigate uncertainty with respect to intrinsic dynamics and variability of the (model) climate system. For this reason, a simple dynamical model is used here with stochastic forcing to represent unresolved processes and to generate a climate that is not a steady equilibrium but a statistical steady state.

A variety of choices must be made in formulating a simple model. In the present paper, one simplification of note is the use of a regional model (as opposed to a global model), with a focus on the descending branch region of the Walker and/or Hadley cells where shallow clouds are most prominent. The regional approach has also been used by, e.g., [21], but for deep convective regions rather than shallow cloud regions, and by, e.g., [2] in a steady, deterministic model for shallow cloud regions but with a hydrological cycle that is not completely free to vary since it must satisfy certain assumed constraints. A more comprehensive approach would be a two-box model or other approach for representing both the ascending and descending branches of the Walker and/or Hadley circulations; such an approach is advantageous because it provides a more complete version of a climate system, although even this approach does not fully represent a complete climate system, and it comes at the expense of greater complexity and a larger number of parameters and parameterizations. For these reasons, a regional model is explored here for a focus on shallow clouds, and interesting questions related to more complete versions of the climate system will be left as future work.

A potentially valuable framework for understanding clouds and climate is the paradigm of phase transitions from statistical physics [44]. To fit this paradigm, the cloud fraction could be taken as an indicator of the "phase" in the transition from a non-cloudy state (phase indicator equals 0 ) to a cloudy state (phase indicator equals 1). If shallow clouds do represent a phase transition in the sense of other systems from statistical physics, then a variety of cloud and climate processes could be viewed as interconnected, providing an umbrella of conceptual understanding. For instance, the cloud fraction, climate uncertainty, and climate sensitivity may all together display singular behavior at the phase transition, analogous to an order parameter (or phase indicator, such as mean magnetization), its variance, and susceptibility in other systems. In the context of shallow clouds, the possibility of a phase transition was suggested by [38], following earlier analyses of phase transitions and criticality in deep convection by [30]. In the present paper, a further step is taken to consider not just the phase indicator (cloud fraction, precipitation, or something similar) but also temperature. What is the behavior of temperature and temperature variance (uncertainty) associated with a phase transition in cloudiness? Such a question is tied to other main questions of this paper-the impact of shallow clouds on temperature and uncertainty-although now phrased in the potentially revealing framework of statistical physics.

The paper is organized as follows. The stochastic model is described in section 2, followed by analyses of its climatological mean state (section 3) its variability/uncertainty (section 4), and climate sensitivity (section 5), with an emphasis on changes that arise as the shallow cloud fraction changes, from non-cloudy to cloudy. Additional physical effects and parameter sensitivities are investigated in section 6 . The connection with the statistical physics concept of phase transitions is then explored in section 7. Conclusions are summarized in section 8 . 


\section{Model Description}

Here we describe an interactive model for temperature and moisture dynamics with cloud-radiative feedbacks. The goal is to have a model that is similar in complexity to the simplest models of radiative equilibrium [as in textbooks such as [31] chap. 6], but in addition incorporating basic moisture and cloud processes, and also stochastic variability.

In this spirit, the model is characterized by three variables: ocean surface temperature $T_{o}$, atmospheric boundary layer temperature $T_{a}$, and total water (vapor plus liquid) integrated from the surface to the top of the boundary layer, $q$. As such, the model consists of two equations for energy balance - one for the ocean surface and one for the boundary layer - and one equation for moisture balance in the boundary layer. The evolution equations are taken to be

$$
\begin{aligned}
\Delta z_{o} c_{o} \rho_{o} \frac{d T_{o}}{d t} & =F_{\text {rad,o }}-\rho_{o} L_{v} E_{s}-F_{\text {sens }}, \\
\Delta z_{a} c_{a} \rho_{a} \frac{d T_{a}}{d t} & =F_{\text {rad }, a}+F_{\text {cloud }}+F_{\text {sens }}+F_{a}, \\
\frac{d q}{d t} & =E_{s}-E_{c}+\xi+F_{q} .
\end{aligned}
$$

The parameters $\Delta z, c, \rho_{a}$, and $\rho_{o}$ represent respectively, the layer thickness, specific heat, and density of the air or water of each layer. Lists of dry and moist parameter values are given in Tables 1 and 2, respectively, and described further in the text below.

Table 1: Parameters associated with 'dry' components of the model.

\begin{tabular}{l|c|r}
\hline parameter & value & Physical Description \\
\hline$S$ & $436 \mathrm{Wm}^{-2}$ & Solar flux at top of free troposphere \\
$\sigma$ & $5.67 \times 10^{-8} \mathrm{Wm}^{-2} \mathrm{~K}^{-4}$ & Stefan-Boltzmann constant \\
$\Delta z_{o}$ & $10 \mathrm{~m}$ & $\begin{array}{r}\text { Ocean surface layer thickness } \\
\Delta z_{a}\end{array}$ \\
$c_{o}$ & $4184 \mathrm{~J} \mathrm{~kg} \mathrm{~m}^{-1} \mathrm{~K}^{-1}$ & Atmospheric cloudy boundary layer thickness \\
$c_{a}$ & $1005 \mathrm{~J} \mathrm{~kg}^{-1} \mathrm{~K}^{-1}$ & Specific heat (ocean) \\
$T_{f}$ & $265 \mathrm{~K}_{0}$ & Specific heat (atmosphere) \\
$\rho_{o}$ & $1000 \mathrm{~kg} \mathrm{~m}^{-3}$ & Free troposphere temperature \\
$\rho_{a}$ & $0.885 \mathrm{~kg} \mathrm{~m}^{-3}$ & Density of ocean water \\
$a_{l 0}$ & 0.24 & Density of boundary layer air \\
$a_{l f}$ & 0.72 & Dry air longwave absorptivity \\
$a_{s}$ & 0.05 & Free troposphere longwave absorptivity \\
$a_{s f}$ & 0.15 & Boundary layer shortwave absorptivity \\
$\tau_{s}$ & 6 days & Free troposphere shortwave absorptivity \\
\hline
\end{tabular}

The source terms are illustrated schematically in Fig. 1 and can be divided into three categories, as described further in the subsections below. First, the moisture and cloud processes (section 2.1) are surface evaporation $E_{s}$, cloud latent heating $F_{c l o u d}$, and cloud-top mixing and evaporation $E_{c}$. Second, the radiative heating/cooling terms (section 2.2) for the ocean and atmospheric boundary layer are $F_{r a d, o}$ and $F_{r a d, a}$, respectively, and they include water vapor feedback and cloud feedback. Third, the non-interactive source terms (section 2.3) are the two constants $F_{a}$ and $F_{q}$ - representing environmental warming and environmental moistening/drying respectively - and the (stochastic) turbulent fluctuations, $\xi$. Finally, a sensible heat term does not fit within these categories, but it is given by

$$
F_{\text {sens }}\left(T_{o}, T_{a}\right)=C_{a} \frac{T_{o}-T_{a}}{\tau_{\text {sens }}},
$$


Table 2: Parameters associated with Cloud and Water Vapor.

\begin{tabular}{l|c|r}
\hline parameter & value & Physical Description \\
\hline$a_{l 1}$ & 0.66 & Cloud/Water vapor enhanced absorptivity \\
$\tau_{e}$ & 6 days & sea surface evaporation time constant \\
$\tau_{t}$ & 6 days & cloud top mixing with free troposphere \\
$q_{f}$ & $10 \mathrm{~mm}$ & Free troposphere water content \\
$A_{c}$ & 0.6 & Cloud top albedo \\
$q_{0}$ & $-260 \mathrm{~mm}$ & Saturation function offset constant \\
$q_{1}$ & $1 \mathrm{~mm} \mathrm{~K}^{-1}$ & Saturation function linear constant \\
$L_{v}$ & $2.4 \times 10^{6} \mathrm{~J} \mathrm{~kg}^{-1}$ & Latent heat of vaporization of water \\
$D_{\star}$ & $0.3 \mathrm{~mm} \mathrm{hr}^{-\frac{1}{2}}$ & Stochastic forcing coefficient \\
\hline
\end{tabular}

where $C_{a}=\Delta z_{a} c_{a} \rho_{a}$. The parameter $\tau_{\text {sens }}$ is the timescale for sensible heat flux, and following [29] it is set equal to the sea surface evaporation time constant, $\tau_{e}$.



Figure 1: Schematic diagram of the physical processes included in the boundary-layer climate model in (1)-(3).

\subsection{Moisture and cloud processes}

The surface evaporation is represented using a standard bulk parameterization of

$$
E_{s}\left(T_{o}, q\right)=\frac{q_{s a t}\left(T_{o}\right)-q}{\tau_{e}} .
$$

While the time scale $\tau_{e}$ is typically taken to be a function of wind speed in many applications of such a bulk formula, its value here is chosen to be 6 days and to be a constant, partly for simplicity and partly because the present framework does not have dynamically active winds; an extended version of the model with dynamically active winds is an interesting future direction. The saturation water vapor $q_{\text {sat }}(T)$ is given by

$$
q_{\text {sat }}(T)=q_{0}+q_{1} T,
$$

where $q_{0}$ and $q_{1}$ are constant parameters. Here, $q_{s a t}$ is similar to the Clausius-Clapeyron relation, which describes the temperature dependence of the saturation vapor pressure, although here it is an empirical formula applied to the boundary-layer column. This linear form of the saturation water vapor is similar to the empirical dependence seen in the observational analysis of [28] for deep convection, except here modified for shallow convection. A temperature dependence of $q_{1}=1.0 \mathrm{~mm} \mathrm{~K}^{-1}$ is used here for boundary layer clouds, 
which is about $25 \%$ of the temperature sensitivity used by [28] for deep convection. The value $q_{0}$ was chosen to be $-260.0 \mathrm{~mm}$ and was determined by setting $q_{\text {sat }}(300 \mathrm{~K})=40.0 \mathrm{~mm}$. The surface evaporation $E_{s}$ also imparts a cooling of $\rho_{o} L_{v} E_{s}$ on the sea surface in (1).

The model presented in this paper is intended to represent a single $\sim 1 \times 1 \mathrm{~km}^{2}$ or $5 \times 5 \mathrm{~km}^{2}$ column within a region of inhomogeneous cloud cover. Therefore, as a simple choice, we represent the cloud state of the system as either having a cloud present or not having a cloud present using a Heaviside cloud indicator function:

$$
\sigma_{c}= \begin{cases}1 & \text { if } q \geq q_{s a t}\left(T_{a}\right) \\ 0 & \text { if } q<q_{\text {sat }}\left(T_{a}\right) .\end{cases}
$$

Hence, a shallow cloud in the boundary layer is present when the boundary-layer total water exceeds the saturation water vapor. While this is a somewhat crude parameterization since it is applied to the boundarylayer-averaged total water, it is in line with the other crude parameterizations that arise in such a simple setup. Although spatial variation is not explicitly captured in this model, the temporal variability of the single column is modeled and provides similar statistical variation. In section 6 , we explicitly model spatial variability with one-dimensional eddy diffusion and find similar behavior as the standard model. Therefore, this paper focuses on the single column model given by (1)-(3) with greater temporal cloud fraction corresponding to greater spatial cloud fraction. The cloud indicator function $\sigma_{c}$ will be used in defining several interactive sources below, such as cloud latent heating and cloud-radiative feedback.

Boundary-layer moisture loss due to evaporation and cloud-top mixing is given by

$$
E_{c}\left(T_{a}, q\right)=\frac{1}{\tau_{t}}\left(q-q_{f}\right) \sigma_{c} .
$$

Notice the factor of $\sigma_{c}$, which signifies that $E_{c}$ is active only if a cloud is present. The time scale $\tau_{t}$ is chosen to be 6 days, which is similar to the value of 4 days estimated by [8]. The free troposphere water vapor, $q_{f}$, was chosen to be $10.0 \mathrm{~mm}$, which is $\sim 25 \%$ of typical boundary-layer $q$ values in this model. The relative value of $q_{f}$ to $q$ was chosen to allow for a simple parameterization of the cloud-top mixing between the relatively moist, cloudy boundary layer and the relatively dry, free troposphere.

Cloud latent heating is defined as

$$
F_{\text {cloud }}\left(T_{o}, T_{a}, q\right)=\rho_{o} L_{v} \sigma_{c} C_{d},
$$

where $C_{d}$ is the rate of condensation of water in the boundary layer. A saturation adjustment scheme is used to specify $C_{d}$. To determine the form of $C_{d}$, we separate total water $q$ into its contributions from water vapor, $q_{v}$, and liquid water, $q_{l}$, so that $q=q_{v}+q_{l}$. By separating the different source terms in (3), the separate evolution equations for water vapor and liquid water can be written as

$$
\begin{aligned}
& \frac{d q_{v}}{d t}=-\sigma_{c} C_{d}+E_{s}+F_{q}+\xi, \\
& \frac{d q_{l}}{d t}=\sigma_{c} C_{d}-\sigma_{c} E_{c} .
\end{aligned}
$$

[Note that equations (10a) and (10b) do not explicitly appear in the model and are mentioned here only to derive $C_{d}$. The form of $C_{d}$ is then found by enforcing a constraint: the water vapor cannot exceed the saturation level. Therefore, if saturation is reached, then the water vapor equals the saturation value and we must have the constraint

$$
\frac{d q_{v}}{d t}=\frac{d q_{s a t}\left(T_{a}\right)}{d t} \quad \text { if } \quad q \geq q_{s a t}\left(T_{a}\right),
$$

until the total water drops below saturation (i.e., until $q<q_{s a t}\left(T_{a}\right)$ ). Applying this constraint to (10a) and solving for $C_{d}$ gives

$$
C_{d}=E_{s}+F_{q}+\xi-\frac{d q_{s a t}\left(T_{a}\right)}{d t} .
$$

Such a formula is commonly used in large-eddy simulations (LES) and cloud-resolving models (CRMs) [12, 13, 16]. Here it is being used with boundary-layer-integrated quantities, $q$ and $T_{a}$, which is outside its range of 
normal use, but allows a simple parameterization that maintains physical consistency between (i) the amount of latent heating and (ii) the amount of water converted between vapor and liquid. To find the influence of $C_{d}$ on temperature evolution, (12) can be used to write the cloud latent heating as

$$
\begin{aligned}
F_{\text {cloud }} & =\rho_{o} L_{v} \sigma_{c}\left[E_{s}+F_{q}+\xi-\frac{d}{d t} q_{\text {sat }}\left(T_{a}\right)\right] \\
& =\rho_{o} L_{v} \sigma_{c}\left[E_{s}+F_{q}+\xi-\frac{d q_{s a t}\left(T_{a}\right)}{d T_{a}} \frac{d T_{a}}{d t}\right] \\
& =\rho_{o} L_{v} \sigma_{c}\left(E_{s}+F_{q}+\xi-q_{1} \frac{d T_{a}}{d t}\right) .
\end{aligned}
$$

Using this form of $F_{\text {cloud }}$, (2) becomes

$$
\Delta z_{a} c_{a} \rho_{a} \frac{d T_{a}}{d t}=\rho_{o} L_{v} \sigma_{c}\left(E_{s}+F_{q}+\xi-q_{1} \frac{d T_{a}}{d t}\right)+F_{r a d, a}+F_{s e n s}+F_{a} .
$$

Gathering the two time derivative terms together on the left hand side, the final form of the evolution equation for $T_{a}$ becomes

$$
\frac{d T_{a}}{d t}=\frac{1}{\Delta z_{a} c_{a} \rho_{a}+\rho_{o} L_{v} \sigma_{c} q_{1}}\left(\rho_{o} L_{v} \sigma_{c}\left(E_{s}+F_{q}+\xi\right)+F_{r a d, a}+F_{s e n s}+F_{a}\right) .
$$

\subsection{Radiative transfer and cloud feedback}

The radiative transfer of the model is designed to be of similar complexity to textbook radiative equilibrium models [e.g., chapter 6 of [31]], although with the added effects of some highly idealized cloud and moisture feedbacks. A summary of the radiative fluxes included in the model is shown in Fig. 2. The mathematical form for each of the fluxes is given in the following paragraphs.

The downward shortwave fluxes are

$$
\begin{aligned}
F_{1} & =S\left(1-a_{s f}\right), \\
F_{2}\left(T_{a}, q\right) & =S\left(1-a_{s f}\right)\left(1-a_{s}\right)\left(1-A_{c} \sigma_{c}\right), \\
F_{3}\left(T_{a}, q\right) & =S\left(1-a_{s f}\right) A_{c} \sigma_{c} .
\end{aligned}
$$

Here, $F_{1}$ represents the solar flux that has passed through the free troposphere and is incident at the top of the boundary layer; $F_{2}$ represents the solar flux incident on the sea surface (so it has not been absorbed in the atmosphere nor reflected by clouds); and $F_{3}$ is the solar radiation that is returned back to the free troposphere due to cloud-top reflection. In (16), $S$ is the solar constant averaged over a diurnal cycle to average over day/night variations in the shortwave solar flux. The parameters $a_{s}$ and $a_{s f}$ represent the shortwave absorptivity of the boundary layer and free troposphere, respectively. These parameters were set to $a_{s}=0.05$ and $a_{s f}=0.15$, which are similar to solar absorptivity values used in simple radiative equilibrium models [chapter 6 of [31]]. The parameter $A_{c}$ is the (boundary-layer) cloud top shortwave albedo; its value was chosen to be 0.6, consistent with estimates of stratocumulus solar albedo [43]. Notice that the cloud-top reflection terms include the product $A_{c} \sigma_{c}$ so that they are only active when a cloud is present $\left(\sigma_{c}=1\right)$.

The longwave fluxes are

$$
\begin{gathered}
F_{4}\left(T_{a}, q\right)=a_{l f} \sigma T_{f}^{4}, \quad F_{5}\left(T_{a}, q\right)=a_{l f}\left(1-a_{l}\right) \sigma T_{f}^{4}, \quad F_{6}\left(T_{a}, q\right)=a_{l} \sigma T_{a}^{4}, \\
F_{7}\left(T_{o}, T_{a}, q\right)=\sigma T_{o}^{4}, \quad F_{8}\left(T_{o}, T_{a}, q\right)=\left(1-a_{l}\right) \sigma T_{o}^{4},
\end{gathered}
$$

where $\sigma$ is the Stefan-Boltzmann constant, and $a_{l}$ and $a_{l f}$ represent the longwave absorptivities of the boundary layer and free troposphere, respectively. In defining the longwave fluxes, we set the emissivity equal to the longwave absorptivity. [Note that $a_{l}$ includes cloud feedback and moisture feedback and is therefore not a constant; see further description in (18) below.] $F_{4}$ represents the longwave radiation emitted from the free 


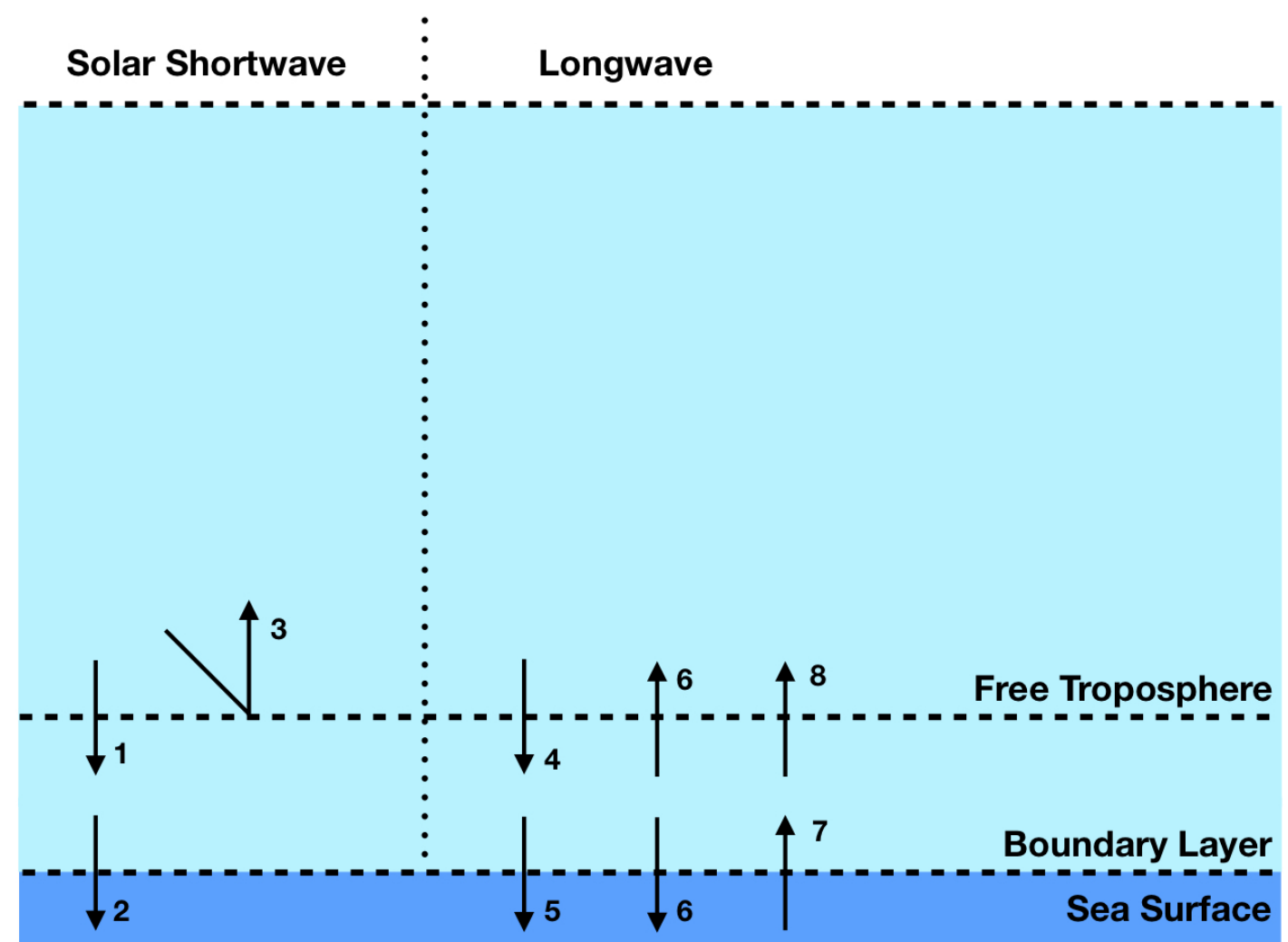

Figure 2: Schematic diagram of the shortwave and longwave fluxes at the sea surface, top of the atmospheric boundary layer, and top of the atmosphere. The number labels correspond to the fluxes in (16)-(17). For instance, $F_{3}$ corresponds to the shortwave flux of solar radiation that passes through the free troposphere and is reflected by clouds at the top of the atmospheric boundary layer.

troposphere, and $F_{5}$ represents the portion of $F_{4}$ that passes through the boundary layer unabsorbed and is incident on the sea surface. $F_{6}$ represents the longwave radiation emitted from the boundary layer. $F_{7}$ represents the longwave radiation emitted from the sea surface. $F_{8}$ represents the portion of $F_{7}$ that passes upward through the boundary layer unabsorbed. This formulation for the longwave emission from the ocean and atmospheric layers is consistent with [31], section 6.4.3.

Cloud feedback and moisture feedback are represented in two ways: through reflection of shortwave radiation (as described above) and also through the boundary layer longwave absorptivity, $a_{l}$. We define this absorptivity with a simple linear function of moisture that is capped at a maximum value upon cloud formation:

$$
a_{l}\left(T_{a}, q\right)=a_{l 0}+a_{l 1}\left[\frac{q}{q_{s a t}}+\sigma_{c}\left(1-\frac{q}{q_{s a t}}\right)\right] .
$$

This linear dependence was chosen because it was the simplest paramterization that captured enhancement of longwave absorptivity due to water vapor. The base, or dry air, absorptivity is represented by $a_{10}$. Longwave absorptivity increases linearly with increasing moisture level relative to saturation and levels off at $a_{l}=a_{l 0}+$ $a_{11}$ when a cloud forms. The base longwave absorptivity was chosen to be $a_{10}=0.24$ and the water vapor enhanced absorptivity was chosen to be $a_{l 1}=0.66$ by setting $a_{l}=a_{l 0}+a_{l 1}=0.9$. The chosen value for $a_{l}$ and the relative values of $a_{l 0}$ and $a_{l 1}$ were motivated by the fact that longwave absorption is dominated by water vapor in the atmosphere, and, upon cloud formation, the boundary layer behaves as a nearly perfect black body in the infrared spectrum. 
In terms of these fluxes, the net radiative flux at the sea surface and boundary layer are given by

$$
\begin{aligned}
& F_{\text {rad,o }}=F_{2}+F_{5}+F_{6}-F_{7}, \\
& F_{\text {rad, } a}=\left(F_{1}-F_{2}-F_{3}\right)+\left(F_{4}-F_{5}\right)+\left(F_{7}-F_{8}\right)-2 F_{6},
\end{aligned}
$$

where a factor of 2 appears in the term $-2 F_{6}$ to account for radiation emitted in two directions (toward space and toward the Earth's surface), consistent with section 6.4 .3 of [31]. These net fluxes, $F_{r a d, o}$ and $F_{r a d, a}$, are the terms that appear in the dynamical model in (1)-(3).

\subsection{External or non-interactive sources}

The source terms $F_{a}, F_{q}$, and $\xi$ are included to represent effects that are not explicitly resolved in the model. For instance, environmental warming $F_{a}$ is a constant parameter, and it represents heat sources that are not explicitly resolved in the model, such as subsiding warm air from the free troposphere or lateral movement of warm air into the column. Environmental drying $F_{q}$ is a similar type of constant parameter, and it represents processes such as lateral movement of moisture out of the column or entrainment into the boundary layer of dry air from the free troposphere. Note that $F_{a}$ and $F_{q}$ have different units, $\mathrm{W} \mathrm{m}^{-2}$ and $\mathrm{mm} \mathrm{day}^{-1}$, respectively, even though we represent both with the same symbol $F$ (with different subscripts), in order to highlight that they play a similar role in the model. The stochastic forcing term $\xi$ represents turbulent fluctuations in moisture sources, and it is represented in idealized form as white noise. Specifically, $\xi=D_{\star} \dot{W}(t)$, where $\dot{W}(t)$ is white noise, and the constant parameter $D \star$ represents the magnitude of the turbulent fluctuations.

\subsection{Setup for numerical simulations}

To generate a climate simulation, the model dynamics in (1)-(3) are numerically integrated using simple standard methods (see section 2.5 for description of numerical methods). The initial conditions for each simulation are given by:

$$
\begin{aligned}
& \left.T_{o}\right|_{t=0}=300 \mathrm{~K}, \\
& \left.T_{a}\right|_{t=0}=290 \mathrm{~K}, \\
& \left.q\right|_{t=0}=25 \mathrm{~mm} .
\end{aligned}
$$

Each simulation was run for a duration of 12 years with a timestep of $\Delta t=0.25$ hours. The final 3 years of data was used for computing statistics to characterize the mean climate state, and the initial 9 years of data were ignored to allow the system to reach statistical equilibrium. While only a few years were typically needed as spin-up to reach statistical equilibrium, 9 years were allowed in order to be conservative.

A suite of over 1000 simulations was carried out in order to assess the impact of different parameters. The main parameters that were varied were the external or non-interactive sources, $F_{a}$ and $F_{q}$. Different $F_{q}$ values were explored from 0 to roughly $-4 \mathrm{~mm} \mathrm{day}^{-1}$ at increments of $0.1 \mathrm{~mm} \mathrm{day}^{-1}$, and $F_{a}$ values were explored from 0 to $50 \mathrm{~W} \mathrm{m^{2 }}$ at increments of approximately $2 \mathrm{~W} \mathrm{~m}^{2}$, for a total of $40 \times 40=1600$ different parameter values. In addition, the longwave absorptivity was also varied as a proxy for carbon dioxide $\left(\mathrm{CO}_{2}\right)$ concentration in section 5, and many other changes to the parameterizations were explored in sections 6 and 7. 


\subsection{Numerical Methods}

To solve (1)-(3) numerically, simple standard methods are used (see e.g., [11, 17]). The equations are discretized using the Euler-Maruyama method:

$$
\begin{aligned}
T_{o}^{n+1}= & T_{o}^{n}+\frac{\Delta t}{\Delta z_{o} C_{o} \rho_{o}}\left(F_{\text {rad,o }}^{n}-\rho_{o} L_{v} E_{s}^{n}-F_{\text {sens }}^{n}\right), \\
T_{a}^{n+1}= & T_{a}^{n}+\frac{\Delta t}{\Delta z_{a} C_{a} \rho_{a}+\rho_{o} L_{v} \sigma_{c} q_{1}}\left[\rho_{o} L_{v} \sigma_{c}\left(E_{s}^{n}+\xi^{n}+F_{q}\right)\right. \\
& \left.\quad+F_{\text {rad,a } a}^{n}+F_{\text {sens }}^{n}+F_{a}\right], \\
q^{n+1}= & q^{n}+\Delta t\left(E_{s}^{n}-E_{c}^{n}+\xi^{n}+F_{q}\right) .
\end{aligned}
$$

Note that (22) is based on (15), the reformulated version of (2). In brief, this method essentially follows a forward Euler discretization, and the stochastic forcing term is interpreted in the Ito sense and is approximated in a non-anticipating way, meaning that the updated temperature and moisture state at time $t_{n+1}=(n+1) \Delta t$ is influenced only by past forcing at time $t_{n}=n \Delta t$. The stochastic forcing term is $\xi(t)=D_{\star} \dot{W}(t)$ in continuum form, and in discretized form it is $\xi^{n} \Delta t=D \star \Delta W^{n}$, which is a Gaussian random variable with mean of 0 and variance of $D_{\star}^{2} \Delta t$, since the variance of the Wiener increment $\Delta W^{n}$ is $\Delta t$. Since $\xi(t)$ is proportional to white noise, the values of $\xi(t)$ at different times are statistically independent (e.g., for time steps $n$ and $m$, the values of $\xi^{n}$ and $\xi^{m}$ with $n \neq m$ are statistically independent in the discrete time version). In practice, this means that a new sample $\xi^{n}$ is drawn from a Gaussian distribution at each time step $n$, independent of all previous samples $\xi^{n-1}, \xi^{n-2}$, etc. For the standard version of the model, we chose a value for the stochastic forcing coefficient $\left(D_{\star}=0.3 \mathrm{~mm} \mathrm{hr}^{-\frac{1}{2}}\right)$ that yielded a climatological standard deviation in the boundary layer temperature of 1-3 Kelvin. We briefly discuss the influence of different values of the noise strength on the cloud fraction in section 7.

Example time series are shown below in section 4. In brief, the stochastic forcing drives a random, turbulent evolution of the moisture. A cloud is formed when the moisture fluctuation rises to the saturation level, and the cloud will dissipate if the moisture sink (evaporation and cloud-top mixing) is strong enough to overcome the moisture sources. At the same time, the saturation water vapor $q_{s a t}\left(T_{a}\right)$ is itself evolving and influencing cloud formation and dissipation.

As one consistency check, we have compared results with the textbook model of [31]. That model uses a single atmospheric layer and applies a globally averaged cloud albedo of 0.3 . To compare this case to our model without moisture, we fixed the cloud indicator to be on $\left(\sigma_{c}=1\right)$ with a cloud albedo of 0.3 and removed all other cloud/moisture dependencies in the model. We also removed the interaction with the free troposphere. With these modifications, our model equilibrated with a sea surface temperature of $T_{o} \approx 295 \mathrm{~K}$ and boundary layer temperature of $T_{a} \approx 255 \mathrm{~K}$ which is comparable to the values from the model in [31].

\section{Shallow-Cloud Impact on Mean Climate}

We now apply this model to simulate the evolution of the atmosphere-ocean system. Since the model in (1)-(3) is highly idealized and similar in complexity to textbook models of radiative equilibrium [chapter 6 of [31]], the model predictions will not be as accurate as comprehensive global climate models that include atmospheric circulations and other complexities. Nevertheless, the trends in the model's climate state can provide useful information, and here we ask: How do shallow clouds impact the mean climate state?

The mean climate state is described in Fig. 3 in terms of temperature and cloud fraction, where we define cloud fraction to be the ratio between the time that clouds are present to the total time (i.e., the final 3 years of simulation time). To explore a variety of environmental conditions, we consider many different values for environmental warming $F_{a}$ and drying $F_{q}$. Changes in these parameters are meant to represent different regions of the tropics and/or subtropics within different parts of the subsiding branch of the Walker and/or Hadley circulation. For larger amounts of environmental warming and/or drying, the system resides in a state 
with relatively low cloud fraction as shown in Fig. 3a. As environmental warming and/or drying is lessened, the cloud fraction gradually increases until reaching a maximum value of about 0.8 . The mean boundary layer temperature displays a similar type of behaviour (Fig. 3b); for larger amounts of environmental warming and/or drying, the temperature is warmer; and as environmental warming and/or drying is lessened, the temperature gradually decreases to a minimum value of about $285 \mathrm{~K}$.

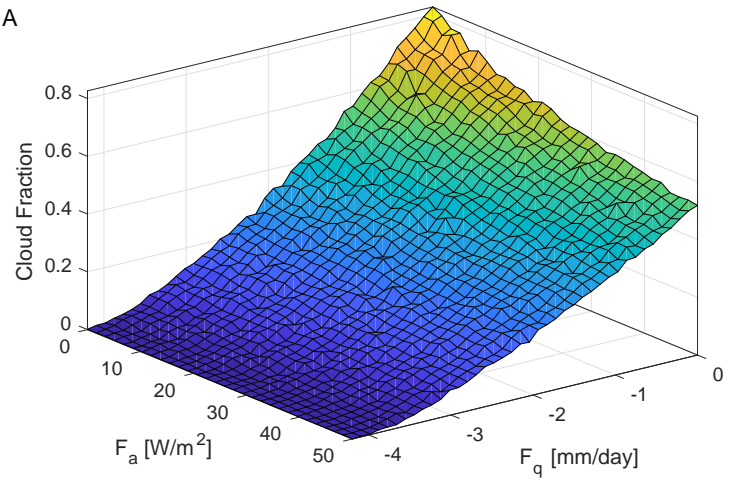

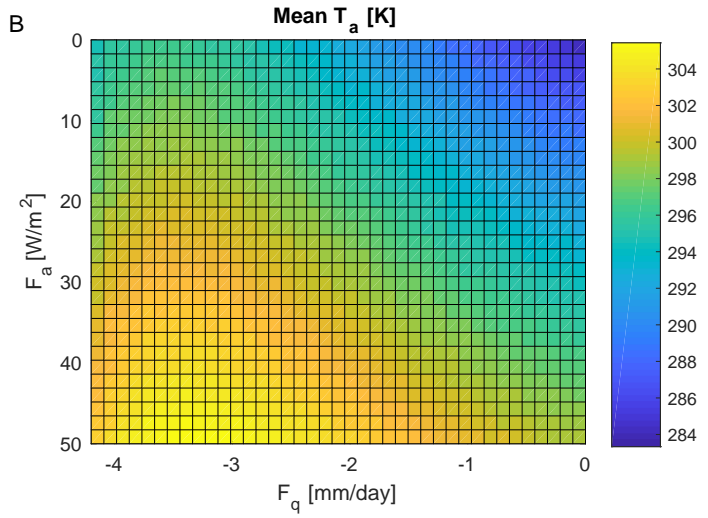

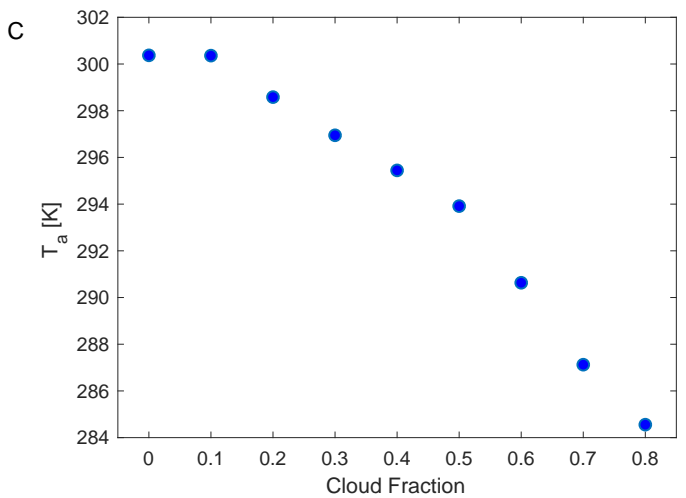

Figure 3: Shallow-cloud impact on mean boundary layer temperature. (A) Cloud fraction as a function of environmental warming $F_{a}$ and drying $F_{q}$. (B) Mean boundary layer temperature as a function of environmental warming and drying. (C) Mean boundary layer temperature as a function of cloud fraction.

To more clearly quantify the impact of shallow clouds on climate, the correspondence between cloud fraction and temperature is shown in Fig. 3c. This figure panel is created by binning the corresponding values of cloud fraction and temperature from Fig. 3a and 3b, and by finding the average temperature value corresponding to each cloud fraction bin. In brief, greater cloud fraction is associated with colder temperature. Physically, the greater cloud fraction causes more solar radiation to be reflected, leading to cooler temperatures; at the same time, lower temperatures cause a lower value of saturation water vapor, thereby further promoting the presence of clouds. This net cooling effect of shallow clouds has also been illustrated in other studies [[24] and references therein], and its appearance here suggests that the simple parameterizations of the present model are somewhat reasonable. Furthermore, note that this mean climate behavior is produced here in a model where a cloud indicator is determined dynamically and interactively, based on dynamically evolving temperature and water content, rather than specifying a prescribed cloud fraction. The (stochastic) dynamical evolution here also allows the study of climate variability, as discussed in subsequent sections. 


\section{Shallow-Cloud Impact on Climate Uncertainty}

Since the present model includes (stochastic) dynamical evolution, it provides not only a mean climate but also climate fluctuations. The fluctuations are a measure of the uncertainty in the mean climate prediction, and here we ask: What is the impact of shallow clouds on climate uncertainty?

To measure the size of the climate fluctuations, the variance in the boundary layer temperature is shown in Fig. 4. In brief, the variance in temperature displays similar behavior to that of mean temperature (Fig. 3). More specifically, for large amounts of environmental warming and/or drying, the system resides in a state with relatively low variance in temperature. As the environmental warming and/or drying is lessened, the variance in the temperature increases.
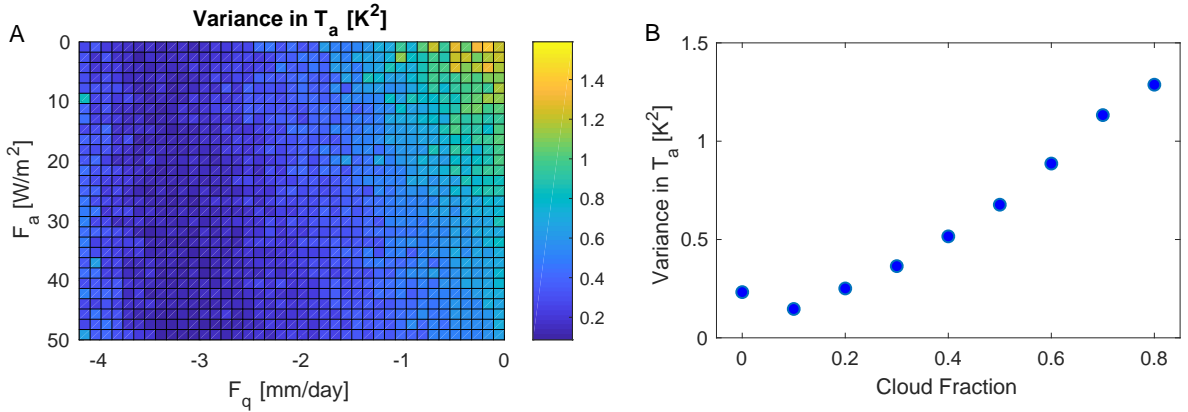

Figure 4: Shallow-cloud impact on variance in boundary layer temperature. (A) Variance in boundary layer temperature as a function of environmental warming $F_{a}$ and drying $F_{q}$. (B) Variance in boundary layer temperature as a function of cloud fraction.

To more clearly quantify the influence of shallow clouds, Fig. 4b shows the correspondence between (i) cloud fraction and (ii) variance in the boundary-layer temperature. This panel is created using the same type of binning procedure that was used earlier in Fig. 3c, except here by binning corresponding values of cloud fraction (Fig. 3a) and temperature variance (Fig. 4a). In short, Fig. 4b shows that greater cloud fraction is associated with higher variance in temperature. In other words, shallow clouds are associated with greater climate uncertainty $[4,6,26]$.

It is the inherent variability of shallow clouds that leads to the model's greater climate uncertainty. To illustrate the switching behaviour of the cloudy state and its impact on atmosphere temperature, figures 5 and 6 show time series plots of several variables for strong and weak environmental drying, respectively. Figure 5 a shows the evolution of the boundary layer temperature with an environmental drying of $-4.0 \mathrm{~mm} /$ day. With strong environmental drying of $-4.0 \mathrm{~mm} /$ day, shown in Figure 5, the boundary layer water content is well below saturation for much of the time series (Fig. 5b), leading to a predominantly cloud free system (Fig. 5c). The inset of figure $5 \mathrm{c}$ shows the cloud events near 0.75 years over a range of 4 days. These rare cloud events persist for up to $\sim 1$ hour. On the other hand, with weak environmental drying of $-0.2 \mathrm{~mm} /$ day, shown in Figure 6, the boundary layer water content fluctuates very near saturation (Fig. 6b), resulting in more frequent cloud events (Fig. 6c). The inset of figure 6c shows the cloud events over the same 4 day span as the inset of figure $5 \mathrm{c}$. The cloud events in the case of weak environmental drying persist over several hours and up to $\sim 1$ day. Due to the succession of cloud events and the associated latent heating, etc., the system undergoes larger variations in the temperature, in comparison to the relatively small temperature variations in the case of Fig. 5 which included few cloud events.

In trying to connect the results of this simple model with global climate change in nature, one simplification of relevance is that this is a local, not global, model over the ocean. Therefore, the model represents the local temperature and local uncertainty. One would expect the local uncertainty to also influence the global uncertainty, through various connections in the climate system, but one would also expect a large local un- 

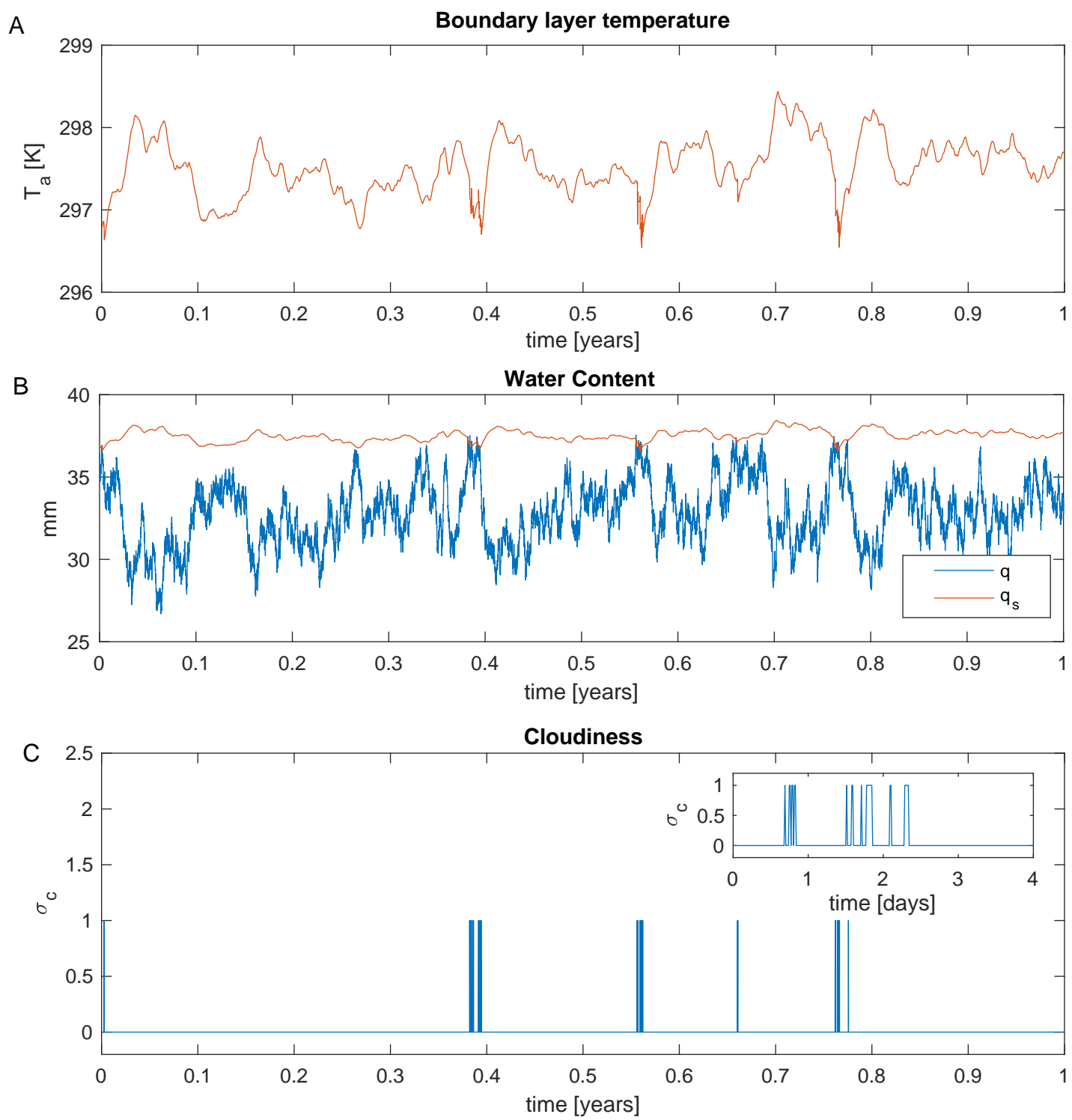

Figure 5: Time series for a case with little occurrence of clouds: $F_{q}=-4.0 \mathrm{~mm} / \mathrm{day}$ and $F_{a}=10.0 \mathrm{~W} / \mathrm{m}^{2}$. (A) Time series for the boundary layer temperature over one year. (B) Time series for the total water $q$ and saturation point $q_{s}$ over one year. (C) Time series for the boundary-layer cloud indicator over one year. (inset) Time series for the cloud indicator over a span of 4 days near the cluster of cloud events near 0.75 years in (C).

certainty to confer a smaller global uncertainty, since the variance of the globally averaged temperature is influenced by shallow cloud regions as well as many other regions of the globe, so the shallow cloud influence would have a smaller impact on the globally averaged temperature. It would be interesting in the future to move beyond the local framework here. As one step in this direction, a spatially varying version of this boundary-layer model is mentioned in section 6 . 

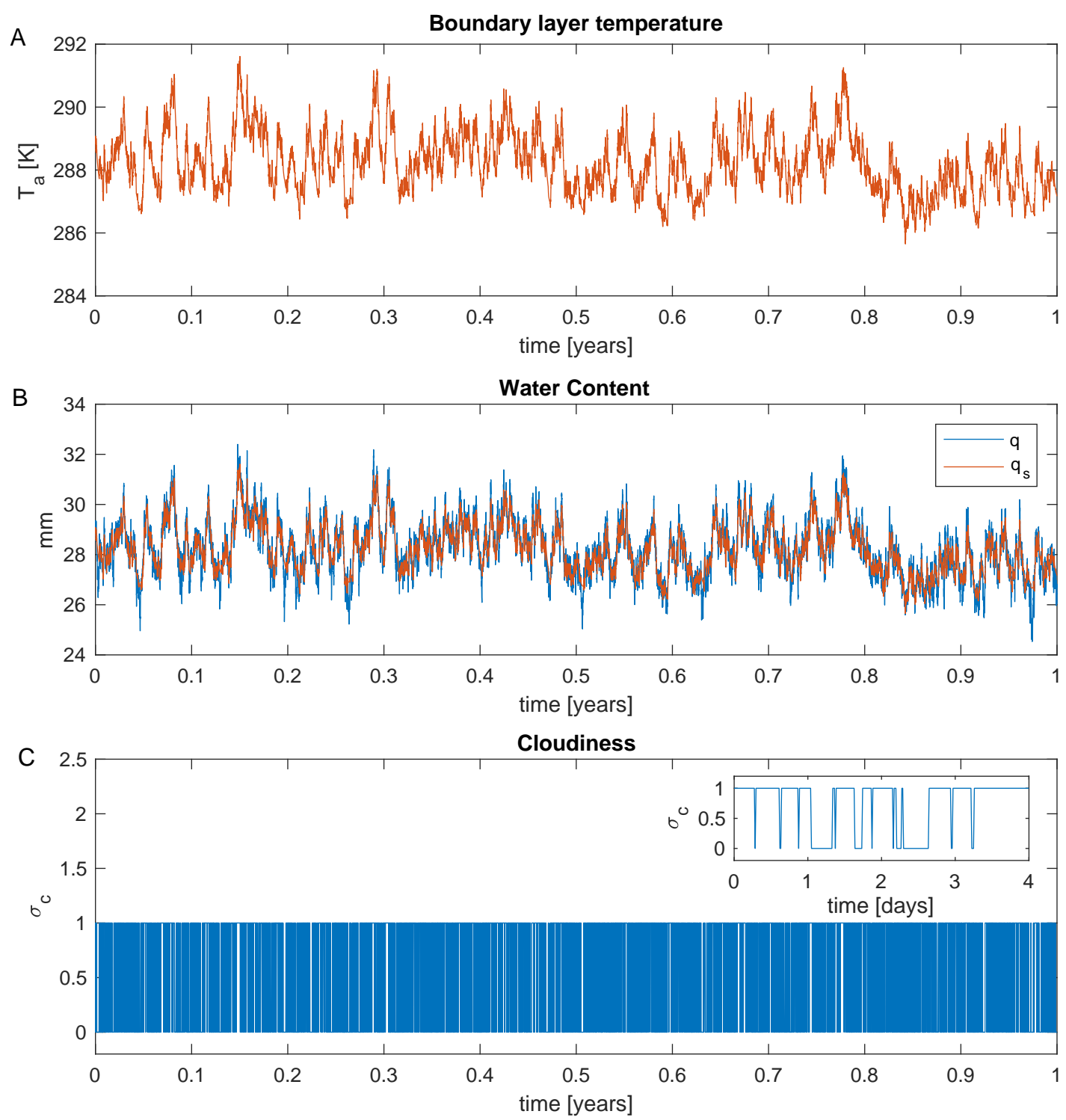

Figure 6: Time series for a system with significant amounts of cloud occurrence: $F_{q}=-0.2 \mathrm{~mm} /$ day and $F_{a}=10.0 \mathrm{~W} / \mathrm{m}^{2}$. (A) Time series for the boundary layer temperature over one year. (B) Time series for the total water $q$ and saturation point $q_{s}$ over one year. (C) Time series for the boundary-layer cloud indicator over one year. (inset) Time series for the cloud indicator over the same 4 day span as the inset in figure $5 c$.

\section{Shallow-Cloud Impact on Climate Sensitivity}

Given the earlier results that show the impact of shallow clouds on mean climate and climate uncertainty, we now investigate whether shallow clouds may have an impact on climate sensitivity. Specifically, we investigate the sensitivity of the mean temperature to changes in atmospheric carbon dioxide, with a particular emphasis on how shallow clouds might influence the sensitivity.

For the setup, as in section 3 we consider the mean climate in terms of cloud fraction and boundary layer temperature, except now over different levels of longwave absorptivity and environmental drying. Changes in the longwave absorptivity are meant to represent variations in atmospheric $\mathrm{CO}_{2}$ over long timescales. Since the model has two longwave absorptivities-one for the boundary layer and one for the free troposphere-we 
vary them both in unison to represent changes in atmospheric $\mathrm{CO}_{2}$ throughout all levels of the atmosphere. In particular, for this analysis we defined a net longwave absorptivity as $1-\left(1-a_{l 0}\right)\left(1-a_{l f}\right)$, to represent the fraction of longwave radiation emitted from the sea surface that would be absorbed by the boundary layer (via $a_{l 0}$ ) or free troposphere (via $a_{l f}$ ) combined. This "net $a_{l}$ " parameter was set by specifiying the dry air transparency factor $\left(1-a_{l 0}\right)\left(1-a_{l f}\right)$ for the combined boundary layer and free troposphere system. To ensure proportionally consistent changes in absorptivity between the boundary layer and free troposphere, we also maintain the ratio $a_{l f} / a_{l 0}=3$, which maintains the same ratio of these parameters that was used in the standard parameter values.

The climate sensitivity results are shown in Figure 7. Broadly speaking, Figures 7a,b show that, as carbon dioxide concentration increases (i.e., as net longwave absorptivity $a_{l}$ increases), the climate warms and becomes less cloudy.
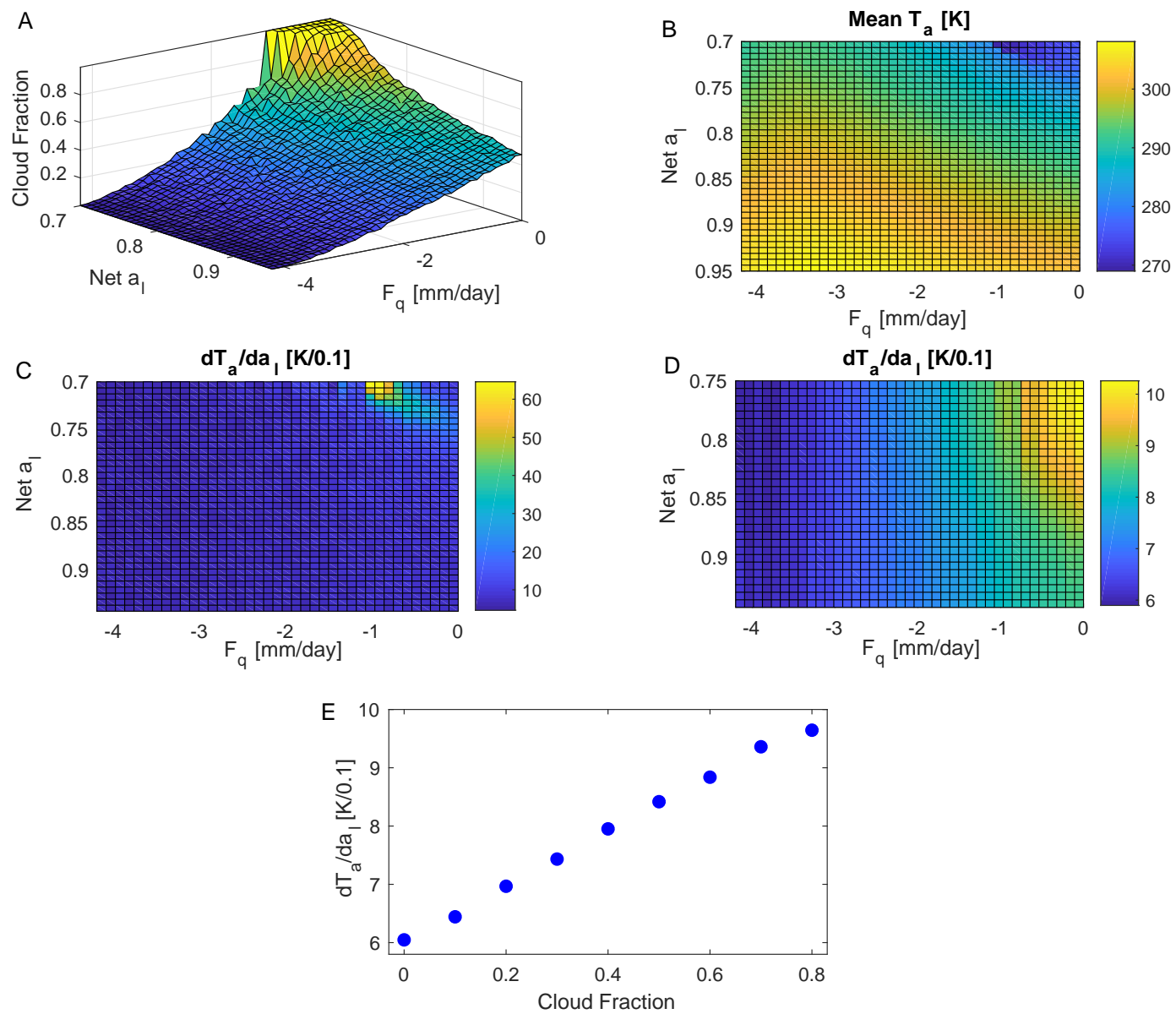

Figure 7: Shallow-cloud impact on climate sensitivity. (A) Cloud fraction as a function of combined longwave absorptivity (Net $a_{l}$ ) from both the boundary layer and free troposphere. (B) Mean boundary layer temperature as a function of environmental drying $\left(F_{q}\right)$ and combined longwave absorptivity. (C) Boundary layer temperature sensitivity to changes in combined longwave absorptivity. (D) Same as (C) except only including Net $a_{l}>0.75$, excluding the region of sharp increase in cloud fraction. (E) Boundary layer temperature sensitivity to changes in combined longwave absorptivity as a function of cloud fraction.

Interestingly, also in Figure 7a,b, a very sharp transition occurs as the absorptivity decreases: the cloud fraction rapidly switches to a regime with $100 \%$ cloud coverage, and the temperature undergoes a rapid cooling. A similar type of climate transition was also recently reported by [37], who investigated a more realistic but still somewhat simplified setup involving a large-eddy simulation (LES) of stratocumulus clouds, coupled with a simple model of the larger-scale circulation. A hysteresis behavior was also reported by [37], and the 
possibility of hysteresis in the present simple model would be interesting to investigate in further detail in the future. (See section 7 for some brief discussion of hysteresis effects.)

Explicit plots are shown in Figures $7 \mathrm{c}, \mathrm{d}$ of the sensitivity of the climate to changes in longwave absorptivity. The sensitivity $d T_{a} / d a_{l}$ was determined by performing a cubic spline fit to the $T_{a}$ data in figure $7 \mathrm{~b}$ and taking the derivative of the fit with respect to the net longwave absorptivity. The climate is most sensitive near the sharp increase in cloud fraction. Away from the sharp increase, the climate sensitivity is lower by an order of magnitude, and it is illustrated more clearly in Figure 7d, which shows the climate sensitivity excluding the region of sharp increase in cloud fraction. For strong amounts of environmental drying, the system has relatively low sensitivity to changes in longwave absorptivity. For weaker amounts of environmental drying, the system becomes more sensitive to changes in longwave absorptivity.

The correspondence between cloud fraction and climate sensitivity is shown in figure 7e. The plot is created using data from figure $7 \mathrm{a}$ and $7 \mathrm{~d}$, which excludes the extremely high sensitivity of figure $7 \mathrm{c}$ and is therefore a conservative estimate. The climate sensitivity is about $60 \%$ greater for a climate with $80 \%$ cloud fraction compared to a climate without clouds. Generally speaking, increasing cloud coverage is associated with increasing climate sensitivity.

\section{Discussion of Additional Processes}

Many processes were not included in (1)-(3), as the model presented in this paper is intended to be similar in complexity to the simplest radiative equilibrium models [chapter 6 of [31]]. With this goal in mind, we chose model (1)-(3) as the standard version after considerable exploration, since it was the simplest version of the model that produced the behaviour discussed in sections 3-5 linking greater cloud fraction to colder temperatures, increased climate variability, and greater climate sensitivity. In developing this model, several additional processes were explored for interesting behaviour. Here we note a few modifications to the proposed model that produced no significant changes.

A sea surface albedo of 0.06 was explored in this model. The low albedo had a negligible effect on sea surface and boundary layer temperature, so it was removed altogether to treat the sea surface as a perfect black-body.

An evolving free troposphere temperature $T_{f}$ was also explored in the model. The free troposphere temperature was interactive only with shortwave cloud feedback and longwave radiation coming from the sea surface and boundary layer. Under this model, a free troposphere thickness of $\Delta z_{f}=14 \mathrm{~km}$ and free tropospheric air density of $\rho_{f}=0.37 \mathrm{~kg} \mathrm{~m}^{-3}$ were used. Mean climate studies with an evolving free troposphere changed very little compared to the standard version with fixed free troposphere temperature.

A temperature-dependent stochastic forcing was initially included in the model to represent greater boundary-layer turbulence at warmer temperatures. This modification had little effect compared to the standard version with fixed stochastic forcing strength.

A time varying solar flux was included in the model to represent day/night variations in the shortwave solar flux. This modification resulted in oscillations in the boundary layer temperature of about 1-2 Kelvin but had no effect on the time averaged statistics of the system.

Drizzle can also potentially influence shallow clouds [36, 45]. To explore in the model, a drizzling indicator was also investigated. The system was able to go between cloudy and drizzling states according to

$$
\begin{array}{rlll}
\sigma_{c}=0 & \text { until } & q \geq q_{\text {sat }}\left(T_{a}\right) \quad \text { and then } \\
\sigma_{\text {drizzle }}=0 & \text { until } & q \geq q_{\text {drizzle }} \\
\sigma_{\text {drizzle }}=1 & \text { until } & q<q_{\text {sat }}\left(T_{a}\right)
\end{array}
$$

A corresponding precipitation term was added to equation (3) and took the form

$$
P=\frac{q-q_{\text {drizzle }}}{\tau_{\text {drizzle }}} \sigma_{\text {drizzle }}
$$


The drizzle time constant was set to $\tau_{\text {drizzle }}=4$ days and the drizzle threshold was given by

$$
q_{\text {drizzle }}=q_{\text {sat }}\left(T_{a}\right)+2 \mathrm{~mm} .
$$

In tests with drizzle, the drizzle threshold $q_{\text {drizzle }}$ was rarely reached and including the possibility of drizzle states in the model had little impact on the mean climate studies compared to the original model with only cloudy and non-cloudy states. It would be interesting in the future to explore alternative representations of drizzle. For instance, one could allow the drizzle to depend on cloud lifetime and to turn on only after cloud lifetime has exceeded a threshold.

The effects of spatial variability were also considered by treating (1)-(3) as the dynamics at an individual grid cell of width $1 \mathrm{~km}$ or $5 \mathrm{~km}$, and by including eddy diffusion as a simple representation of spatially varying turbulence effects. Such a setup is similar to [38], except with more detailed representations of different physical processes here. Some initial cases were explored with one-dimensional variability in $x$ but no variability in the $y$ direction, and they produced essentially the same types of behavior as the standard version of the model without spatial variability. It would be interesting in the future to further explore spatially variations or coupling with the larger-scale circulation in some way.

The surface here was treated as an ocean surface, described by temperature $T_{0}$. It could be interesting in the future to investigate a similar model over land, in which case it may also be appropriate to include a second surface variable to represent soil moisture.

\section{Phase Transition}

A very abrupt transition was seen earlier in Figure 7: the cloud fraction and temperature changed rapidly as the carbon dioxide concentration (i.e., longwave absorptivity) and environmental drying were varied. This behavior is reminiscent of a phase transition, and in this section we explore the question: To what extent are phase transitions seen in this model of shallow clouds and climate?

As background, phase transitions are seen in a variety of contexts in statistical physics, such as magnetism (with two phases-spin-up and spin-down) and water (with three phases-solid, liquid, and gas) [44]. It has been proposed that shallow clouds may also exhibit phase transitions, where the two phases could be open-cell and closed-cell stratocumulus phases, and/or cloudy and non-cloudy phases as indicated by cloud fraction [22, 38]. Other possible phase transitions related to deep convection have also been proposed and investigated in some detail $[1,18,19,28,30,39,40]$. For shallow clouds, the present model provides an opportunity to look for phase transitions and to study the relation with many atmospheric quantities of interest, such as cloud fraction, temperature, and environmental factors. This more detailed view could motivate observational studies and indicate different ways of analyzing conditional statistics in observational data, along the lines of the analyses of [42] and [10] and references therein.

To investigate the possibility of phase transitions in the model, we considered a variety of possible parameter values. In the standard parameter values of Figures 3-6, no sharp transitions were seen over the range of environmental warming and drying parameters shown. Nevertheless, beyond the standard parameter values, it was shown in Figure 7 that sharp variations reminiscent of phase transitions could occur for different values of the $\mathrm{CO}_{2}$ concentration/longwave absorptivity.

Cloud albedo was also found to be a controlling factor for the presence or absence of phase transitions. Figure 8 shows the mean climate state for a larger value of 0.7 for the cloud albedo, in contrast to the standard value of 0.6. Many of the general trends from the case of Figure 3 can also be seen in Figure 8, except a new feature arises: a discontinuous jump appears in the cloud fraction and temperature, as the environmental forcing is varied, similar to a phase transition. Another feature of phase transitions is seen in Figure 9 in the temperature variance: the jump in mean temperature is accompanied by a spike in the variance. In other words, the climate uncertainty has a pronounced spike at the border between the two climate regimes. Furthermore, while the variance is still typically increasing as cloud fraction increases (i.e., climate uncertainty is associated with shallow clouds), a new feature appears in this scenario with a phase transition in Figure 9c: 
the maximum climate uncertainty is associated with a climate with roughly $70 \%$ cloud fraction, and climate uncertainty slightly decreases for higher cloud fractions. Such behavior is consistent with the occurrence of a completely cloudy climate state (cloud fraction equals 1), which has lower uncertainty due to its lack of variability from cloud formation and decay. Finally, climate sensitivity shows a similar type of behavior as climate uncertainty. Specifically, in Figure 10, when carbon dioxide concentration/longwave absorptivity is taken as a control parameter, a similar phase transition is seen, where the climate state changes abruptly and a spike in the climate sensitivity occurs at certain $\mathrm{CO}_{2}$ concentrations.

A

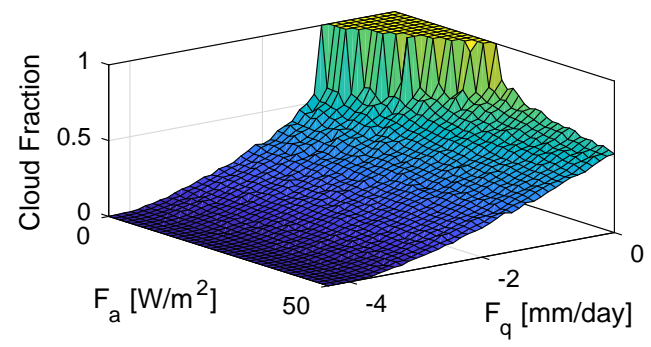

C

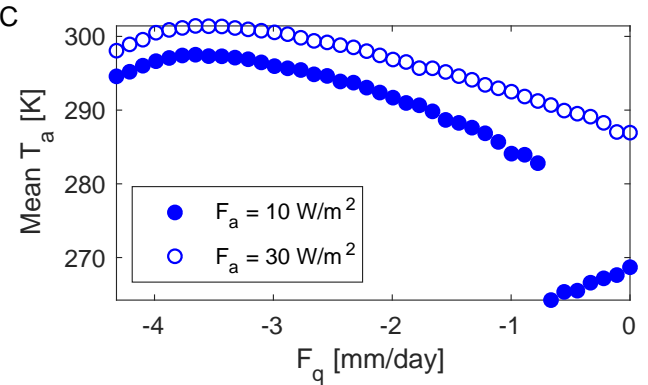

B


Figure 8: Mean climate for a system with increased cloud albedo of $A_{c}=0.7$. (A) Cloud fraction as a function of environmental warming and drying. (B) Mean boundary layer temperature as a function of environmental warming and drying. Note the different temperature range compared with Fig. 3. (C) Line cuts through (B) for $F_{a}=10 \mathrm{~W} / \mathrm{m}^{2}$ (filled circles) and $F_{a}=30 \mathrm{~W} / \mathrm{m}^{2}$ (open circles). (D) Mean boundary layer temperature as a function of cloud fraction.

What is the underlying source of the phase transition or climate regimes in this model? One possibility is that it is related to multiple climate equilibria. To investigate this possibility, we analyzed the deterministic version of the model in (1)-(3) and looked for steady-state solutions. Since the dynamical model is only allowed to be completely cloudy $\left(\sigma_{c}=1\right)$ or non-cloudy $\left(\sigma_{c}=0\right)$, we also considered a smoothed version of the model, where the Heaviside function that defines $\sigma_{c}$ in (7) is smoothed out as a piecewise-linear continuous function; consequently, the model is allowed to enter into a partially cloudy steady state with any value of $\sigma_{c}$ between 0 and 1 . The equilibria are illustrated in Figure 11. In brief, we find that the model appears to have multiple steady states for certain parameter values, in a way that is consistent with the different climate regimes seen in Figures 3-10. For instance, when a partially cloudy climate state is seen, it is often the case that a completely cloudy equilibrium state also exists; but the initial conditions in (20) were more conducive to reaching the partially cloudy climate equilibrium. In fact, using colder initial conditions allowed the system to equilibrate into the completely cloudy state for all values of $F_{q}$ and $F_{a}$. It would be interesting in the future to investigate different experimental setups that would allow the possibility of hysteresis, as seen by, e.g., [37] in other types of models. In the present model, along with these two stable or attracting equilibria, a third equilibrium state is also often present, and it appears to be unstable. A bifurcation appears to occur when the "plateau" arises in Figures 8-10: two of the three equilibria disappear, and the completely cloudy state remains as the only equilibrium state. 

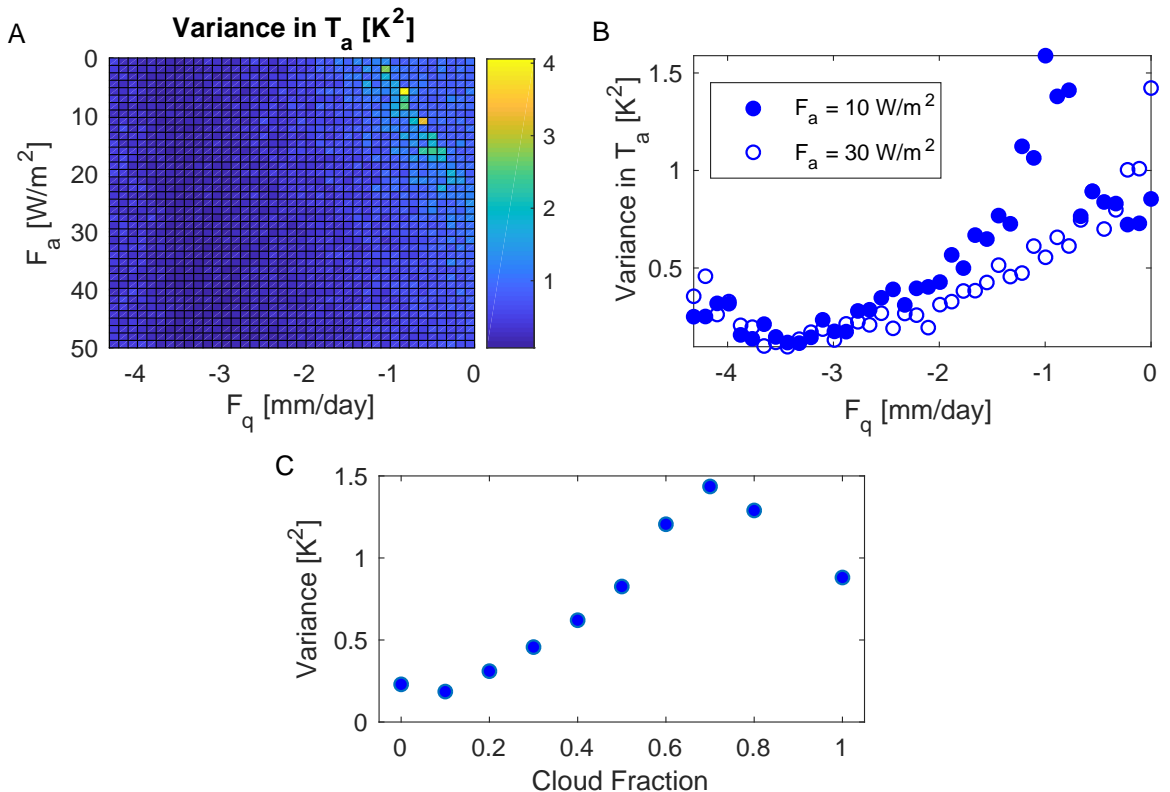

Figure 9: Variance in boundary layer temperature for a system with increased cloud albedo of $A_{c}=0.7$. (A) Variance in boundary layer temperature as a function of environmental warming and drying. (B) Line cuts through (A) for $F_{a}=10 \mathrm{~W} / \mathrm{m}^{2}$ (filled circles) and $F_{a}=30 \mathrm{~W} / \mathrm{m}^{2}$ (open circles). (C) Variance in boundary layer temperature as a function of cloud fraction.
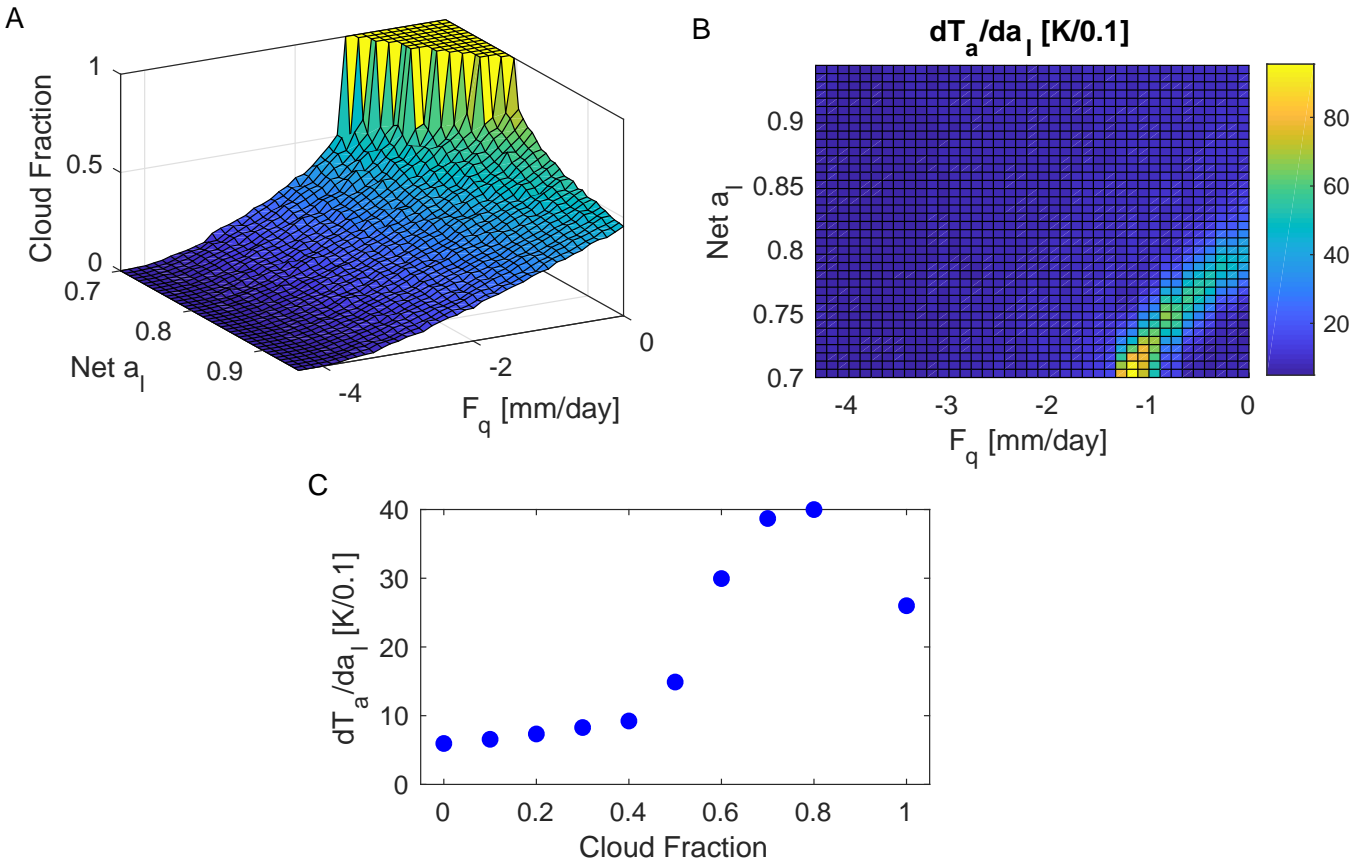

Figure 10: Climate sensitivity to changes in longwave absorptivity for a system with environmental warming of $F_{a}=20 \mathrm{~W} / \mathrm{m}^{2}$ and increased cloud albedo of $A_{c}=0.7$. (A) Cloud fraction as a function of net longwave absorptivity and environmental drying. (B) Boundary layer temperature sensitivity to changes in combined longwave absorptivity. (C) Boundary layer temperature sensitivity to changes in combined longwave absorptivity as a function of cloud fraction.

In addition to this deterministic analysis, it is also interesting to see the influence of the noise strength. In particular, as shown in Figure 12, the completely cloudy climate regime can be eroded if the stochastic noise (i.e., the turbulent variability) is larger. The larger noise allows for excursions out of the completely cloud 

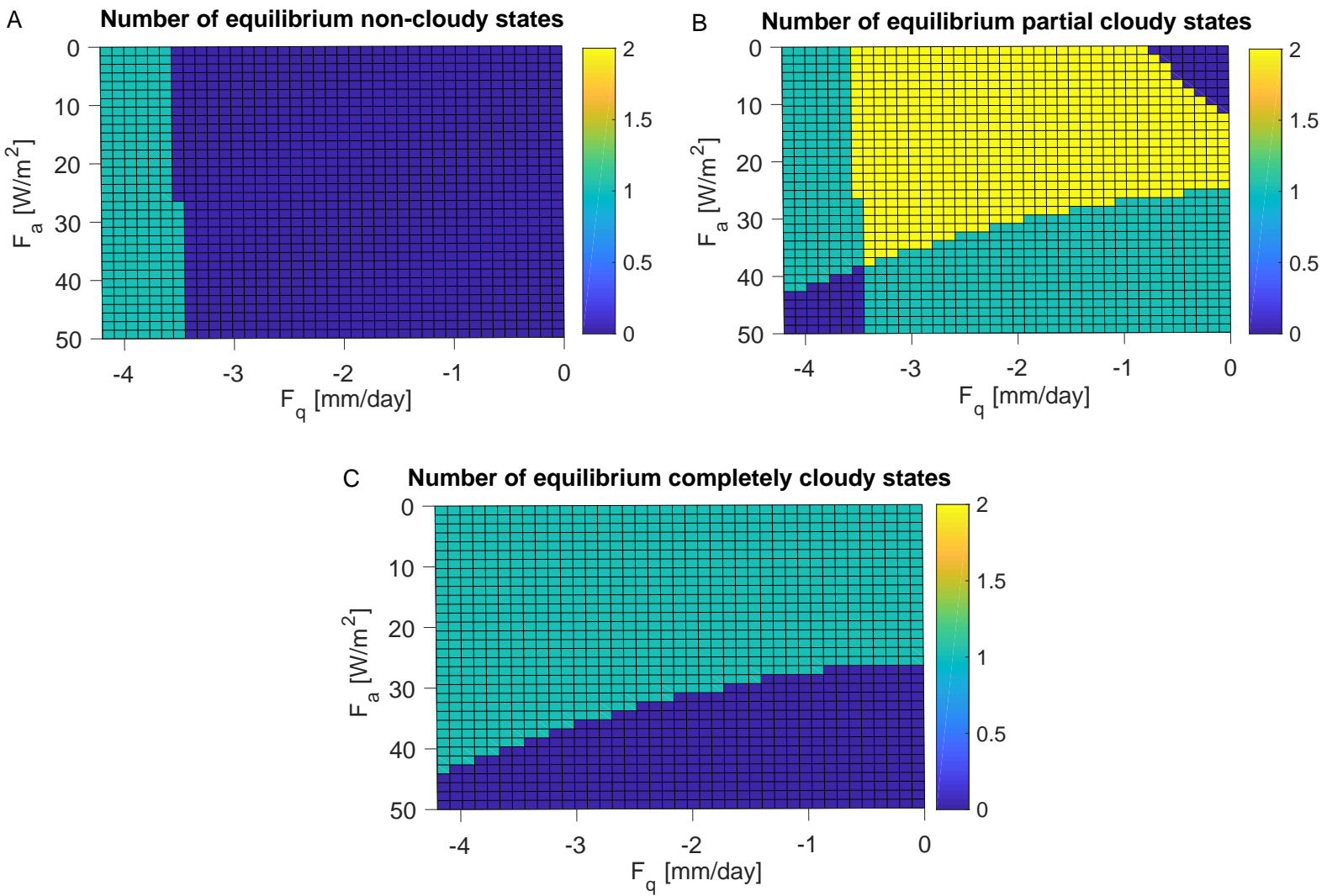

Figure 11: (A) Number of non-cloudy equilibrium states $\left(\sigma_{c}=0\right)$. (B) Number of equilibrium states with partial cloudiness $(0<$ $\left.\sigma_{c}<1\right)$. (C) Number of equilibrium states with complete cloudiness $\left(\sigma_{c}=1\right)$.

state, so the system does not stay locked into the completely cloudy climate state forever. This dependence on noise strength is also reminiscent of phase transitions in other contexts [44]; for instance, in magnetic systems, the temperature is the indicator of the strength of system noise, and it is the high-temperature case that lacks the ordered magnetic state (spin 1), analogous to a high-turbulence case that lacks the completely cloudy state (cloud fraction 1) in the simple climate model.

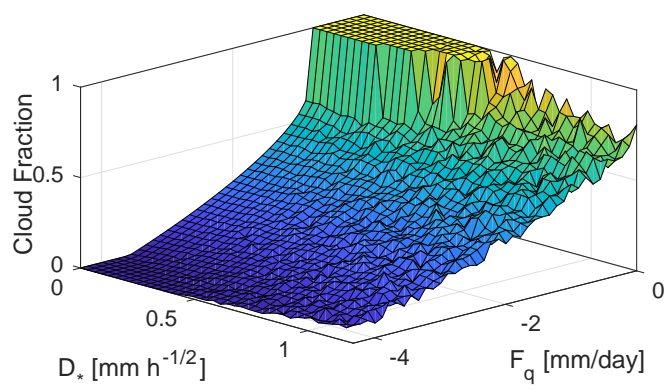

Figure 12: Cloud fraction as a function of noise strength $D_{\star}$ and $F_{q}$ for a system with $F_{a}=10 \mathrm{~W} / \mathrm{m}^{2}$ and an increased cloud albedo of 0.7 . 


\section{Conclusions}

To summarize, our main goal was to investigate how much climate uncertainty arises from the intrinsic dynamics and variability of shallow clouds. A simple stochastic model was formulated to investigate this question, and the model was aimed at the level of complexity of textbook models of radiative equilibrium, except here including dynamical evolution and a statistical steady state instead of a steady equilibrium.

Significantly higher climate uncertainty was seen for a cloudy climate compared with a non-cloudy climate. A significant source of the inherent variability was the off-and-on switching of cloud formation and decay, beyond the ever-present turbulent variability (represented as stochastic forcing) that is the main source of variability in the absence of clouds.

The model here is highly idealized, and it would be interesting in the future to explore additional realism. For example, several additional physical processes were investigated and described in section 6, such as dynamical evolution of the free troposphere, sea-surface-temperature-dependent stochastic forcing, drizzle, and spatial variations. It would also be interesting to consider additional layers of complexity such as a dynamic height of the boundary layer, or to couple this shallow-cloud model with the large-scale circulation in some way, such as a two-box model setup.

Phase transitions in the climate state were seen in the model, where cloud cover and temperature undergo abrupt changes as certain environmental parameters change. One value of the concept of phase transitions is in providing organizing principles. Specifically, it provides a conceptual umbrella that links together a variety of statistics and behaviors (such as climate state transitions and associated increases in climate sensitivity and uncertainty/variance). For deep convection, such a perspective of phase transitions has been investigated in the past [28,30], encompassing many aspects, such as precipitation, water vapor, atmospheric temperature, sea surface temperature, etc. For shallow clouds, the present model provides an opportunity to look for phase transitions and to study the relation with many atmospheric quantities of interest, such as cloud fraction, temperature, and environmental factors. The model results could motivate observational studies and indicate different ways of analyzing conditional statistics in observational data, along the lines of the analyses of [42] and [10] and references therein. Given the large number of possible statistics and viewpoints of clouds and climate, the unifying perspective of statistical physics could be helpful.

Acknowledgments: The authors thank T. L'Ecuyer, A. Douglas, and two anonymous reviewers for helpful discussion. The research of S.N.S. is partially supported by a Sloan Research Fellowship from the Alfred P. Sloan Foundation and a Vilas Associates Award from the University of Wisconsin-Madison.

\section{References}

[1] T. H. Abbott, S. N. Stechmann, and J. D. Neelin. Long temporal autocorrelations in tropical precipitation data and spike train prototypes. Geophys. Res. Lett., 43(21):11-472, 2016.

[2] A. K. Betts and W. Ridgway. Coupling of the radiative, convective, and surface fluxes over the equatorial Pacific. J. Atmos. Sci., 45(3):522-536, 1988.

[3] A. K. Betts and W. Ridgway. Climatic equilibrium of the atmospheric convective boundary layer over a tropical ocean. J. Atmos. Sci., 46(17):2621-2641, 1989.

[4] Peter N Blossey, Christopher S Bretherton, Minghua Zhang, Anning Cheng, Satoshi Endo, Thijs Heus, Yangang Liu, Adrian P Lock, Stephan R de Roode, and Kuan-Man Xu. Marine low cloud sensitivity to an idealized climate change: The cgils les intercomparison. Journal of Advances in Modeling Earth Systems, 5(2):234-258, 2013.

[5] S. Bony, R. Colman, V. M. Kattsov, R. P. Allan, C. S. Bretherton, J.-L. Dufresne, A. Hall, S. Hallegatte, M. M. Holland, W. Ingram, et al. How well do we understand and evaluate climate change feedback processes? J. Climate, 19(15):3445-3482, 2006.

[6] S. Bony and J.L. Dufresne. Marine boundary layer clouds at the heart of tropical cloud feedback uncertainties in climate models. Geophys. Res. Lett, 32:20806, 2005.

[7] Sandrine Bony, Bjorn Stevens, Dargan MW Frierson, Christian Jakob, Masa Kageyama, Robert Pincus, Theodore G Shepherd, Steven C Sherwood, A Pier Siebesma, Adam H Sobel, et al. Clouds, circulation and climate sensitivity. Nature Geoscience, 
8(4):261, 2015.

[8] C. S. Bretherton, P. Austin, and S. T. Siems. Cloudiness and marine boundary layer dynamics in the ASTEX Lagrangian experiments. Part II: Cloudiness, drizzle, surface fluxes, and entrainment. J. Atmos. Sci., 52(16):2724-2735, 1995.

[9] R. D. Cess, G. L. Potter, J. P. Blanchet, G. J. Boer, A. D. Del Genio, M. Deque, V. Dymnikov, V. Galin, W. L. Gates, S. J. Ghan, et al. Intercomparison and interpretation of climate feedback processes in 19 atmospheric general circulation models. J. Geophys. Res., 95(D10):16601-16615, 1990.

[10] Alyson Douglas and Tristan L'Ecuyer. Quantifying variations in shortwave aerosol-cloud-radiation interactions using local meteorology and cloud state constraints. Atmospheric Chemistry and Physics, 19(9):6251-6268, 2019.

[11] C. W. Gardiner. Handbook of stochastic methods: for physics, chemistry \& the natural sciences, volume 13 of Springer Series in Synergetics. Springer-Verlag, Berlin, 2004.

[12] W. W. Grabowski. Representation of turbulent mixing and buoyancy reversal in bulk cloud models. J. Atmos. Sci., 64(10):3666-3680, 2007.

[13] Wojciech W Grabowski and Piotr K Smolarkiewicz. Monotone finite-difference approximations to the advectioncondensation problem. Monthly Weather Review, 118(10):2082-2098, 1990.

[14] D. L. Hartmann, M. E. Ockert-Bell, and M. L. Michelsen. The effect of cloud type on Earth's energy balance: Global analysis. J. Climate, 5(11):1281-1304, 1992.

[15] Dennis L. Hartmann, Leslie A. Moy, and Qiang Fu. Tropical convection and the energy balance at the top of the atmosphere. J. Climate, 14:4495-4511, 2001.

[16] G. Hernandez-Duenas, A. J. Majda, L. M. Smith, and S. N. Stechmann. Minimal models for precipitating turbulent convection. J. Fluid Mech., 717:576-611, 2013.

[17] D.J. Higham. An algorithmic introduction to numerical simulation of stochastic differential equations. SIAM Review, 43(3):525-546, 2001.

[18] S. Hottovy and S. N. Stechmann. A spatiotemporal stochastic model for tropical precipitation and water vapor dynamics. J. Atmos. Sci., 72:4721-4738, 2015.

[19] S. Hottovy and S. N. Stechmann. Threshold models for rainfall and convection: Deterministic versus stochastic triggers. SIAM J. Appl. Math., 75:861-884, 2015.

[20] M. A. Kelly and D. A. Randall. A two-box model of a zonal atmospheric circulation in the tropics. J. Climate, 14(19):39443964, 2001.

[21] M. A. Kelly, D. A. Randall, and G. L. Stephens. A simple radiative-convective model with a hydrological cycle and interactive clouds. Q. J. Roy. Meteorol. Soc., 125(555):837-869, 1999.

[22] Boualem Khouider and Alexander Bihlo. A new stochastic model for the boundary layer clouds and stratocumulus phase transition regimes: Open cells, closed cells, and convective rolls. Journal of Geophysical Research: Atmospheres, 124(1):367-386, 2019

[23] J. T. Kiehl. On the observed near cancellation between longwave and shortwave cloud forcing in the tropical regions. J. Climate, 7:559-565, 1994.

[24] Stephen A Klein and Dennis L Hartmann. The seasonal cycle of low stratiform clouds. Journal of Climate, 6(8):1587-1606, 1993.

[25] K. Larson, D.L. Hartmann, and S.A. Klein. The role of clouds, water vapor, circulation, and boundary layer structure in the sensitivity of the tropical climate. Journal of Climate, 12(8):2359-2374, 1999.

[26] Isabel L McCoy, Robert Wood, and Jennifer K Fletcher. Identifying meteorological controls on open and closed mesoscale cellular convection associated with marine cold air outbreaks. Journal of Geophysical Research: Atmospheres, 122(21):11678, 2017.

[27] R.L. Miller. Tropical thermostats and low cloud cover. Journal of Climate, 10(3):409-440, 1997.

[28] J. D. Neelin, O. Peters, and K. Hales. The transition to strong convection. J. Atmos. Sci., 66(8):2367-2384, 2009.

[29] J. David Neelin and Ning Zeng. A quasi-equilibrium tropical circulation model-formulation. J. Atmos. Sci., 57:1741-1766, 2000.

[30] O. Peters and J. D. Neelin. Critical phenomena in atmospheric precipitation. Nature Physics, 2(6):393-396, 2006.

[31] G. W. Petty. A first course in atmospheric radiation. Sundog Pub, Madison, Wisconsin, USA, 2006.

[32] R.T. Pierrehumbert. Thermostats, radiator fins, and the local runaway greenhouse. J. Atmos. Sci., 52(10):1784-1806, 1995.

[33] V. Ramanathan, R. D. Cess, E. F. Harrison, P. Minnis, B. R. Barkstrom, E. Ahmad, and D. Hartmann. Cloud-radiative forcing and climate: Results from the Earth Radiation Budget Experiment. Science, 243(4887):57-63, 1989.

[34] N. O. Renno, K. A. Emanuel, and P. H. Stone. Radiative-convective model with an explicit hydrologic cycle: 1. Formulation and sensitivity to model parameters. J. Geophys. Res.: Atmos., 99(D7):14429-14441, 1994.

[35] E. S. Sarachik. Tropical sea surface temperature: An interactive one-dimensional atmosphere-ocean model. Dyn. Atmos. Oceans, 2(5):455-469, 1978.

[36] V. Savic-Jovcic and B. Stevens. The structure and mesoscale organization of precipitating stratocumulus. J. Atmos. Sci., 65(5):1587-1605, 2008.

[37] Tapio Schneider, Colleen M Kaul, and Kyle G Pressel. Possible climate transitions from breakup of stratocumulus decks under greenhouse warming. Nature Geoscience, 12(3):163, 2019.

[38] S. N. Stechmann and S. Hottovy. Cloud regimes as phase transitions. Geophys. Res. Lett., 43:6579-6587, 2016. 
[39] S. N. Stechmann and J. D. Neelin. A stochastic model for the transition to strong convection. J. Atmos. Sci., 68:2955-2970, 2011.

[40] S. N. Stechmann and J. D. Neelin. First-passage-time prototypes for precipitation statistics. J. Atmos. Sci., 71:3269-3291, 2014.

[41] G. L. Stephens. Cloud feedbacks in the climate system: A critical review. J. Climate, 18(2):237-273, 2005.

[42] K. D. Williams and M. J. Webb. A quantitative performance assessment of cloud regimes in climate models. Climate Dynamics, 33(1):141-157, 2009.

[43] R. Wood. Stratocumulus clouds. Mon. Wea. Rev., 140(8):2373-2423, 2012.

[44] J. M. Yeomans. Statistical mechanics of phase transitions. Oxford University Press, 1992.

[45] Xiaoli Zhou, Thijs Heus, and Pavlos Kollias. Influences of drizzle on stratocumulus cloudiness and organization. Journal of Geophysical Research: Atmospheres, 122(13):6989-7003, 2017. 